\title{
Host preference of Trichogramma dendrolimi (Hymenoptera: Trichogrammatidae) on its native host, Mamestra brassicae (Lepidoptera: Noctuidae) after 12 continuous generations on a factitious host
}

\author{
Yoichi Takada, ${ }^{1}$ Satoshi Kawamura and Toshiharu Tanaka ${ }^{2, *}$ \\ Gifu Prefectural Institute for Bio-Industrial Technology, Gifu 505-0004, Japan \\ ${ }^{2}$ Laboratory of Applied Entomology, Graduate School of Bio-Agricultural Sciences, Nagoya University, Nagoya 464-8601, Japan \\ (Received 2 June 2000; Accepted 17 November 2000)
}

\begin{abstract}
Factitious hosts are needed for mass-propagation in crop pest management. Egg parasitoids are considered as effective biocontrol agents of lepidopterous pests because they kill host eggs and can usually be easily mass propagated on factitious hosts. However, previous studies indicated that parasitoids which had been reared on a factitious host changed host preference and did not parasitize the native host. In the present report, Trichogramma dendrolimi were collected from egg masses of Mamestra brassicae in cabbage fields maintained without insecticides. T. dendrolimi was maintained in the laboratory for 12 successive generations on Ephestia kuehniella. After the 12 successive generations, the parasitoid females still preferred $M$. brassicae to E. kuehniella for oviposition. T. dendrolimi, that emerged from $M$. brassicae eggs were larger in size and laid twice as many eggs as those from E. kuehniella during their lifetime. These results suggest that $T$. dendrolimi parasitizes the native host without losing host preference even after 12 successive generations on a factitious host.
\end{abstract}

Key words: Biological control, host preference, Trichogramma dendrolimi

\section{INTRODUCTION}

Chalcidoid wasps of the genus Trichogramma are extensively used as biological control agents because they attack eggs of several lepidopterous species that cause serious damage to many kinds of crops (Hassan et al., 1988; Ciochia, 1991; Nikonov et al., 1991; Greenberg et al., 1998a). In inoculative or augmentative release programs for the control of pests, natural enemies should be mass propagated (Stinner, 1977; Sun et al., 1990). In many cases, factitious hosts have been widely used for the mass production of Trichogramma species to reduce costs. However, mass-rearing programs have often resulted in adaptation of the insect to laboratory conditions. Changes in oviposition preference of parasitoids after rearing on factitious hosts have been reported. Van Bergeijk et al. (1989) reported that Trichogramma maidis had reduced acceptance to its natural host eggs of the European corn borer, after 3 generations of continuous rearing on eggs of the Mediterranean flour moth, E. kuehniella. Bautista and Harris (1997) also reported that insectary rearing of Diachasmimorpha longicaudata for $>160$ generations may modify the parasitization behavior.

Trichogramma dendrolimi Matsumura is a candidate for an effective agent to control the population density of lepidopterous pests on cruciferous crops (Sun et al., 1990; Hamada, 1992; Hirai et al., 1996). In this study, we examined whether or not the oviposition preference of $T$. dendrolimi against Mamestra brassicae eggs changes after successive rearing for 12 generations on E. kuehniella eggs, and the allocation of the number of progeny on two different host species, as well as sex allocation in relation to age of the female wasp.

\section{MATERIALS AND METHODS}

Host. M. brassicae were collected in cabbage fields maintained without insecticides at Kagamihara, Gifu Prefecture. A stock culture was continuously maintained using an artificial diet (Insecta LF $^{\circledR}$, Nihon-Nohsan, Kanagawa) under a $16 \mathrm{~L}: 8 \mathrm{D}$ photoperiod at $25 \pm 2{ }^{\circ} \mathrm{C}$. Ten each of newly

\footnotetext{
* To whom correspondence should be addressed.

${ }^{1}$ Present address: Gifu Prefectural Agricultural College, Gifu 509-0241, Japan
} 
emerged male and female adult moths were put in a plastic cylinder $(16 \mathrm{~cm}$ in diameter $\times 20 \mathrm{~cm}$ in height), the inside of which was covered with a thick paper, and fed on 5\% sucrose solution. The egg-mass attached on the inside paper was cut off with a suitable size of paper and used for experiments. A stock culture of E. kuehniella was also continuously reared using wheat flour under the same conditions as described above. The eggs of $E$. kuehniella were trapped on a $250 \mu \mathrm{m}$ mesh after being passed through a $500 \mu \mathrm{m}$ mesh, then attached on a thick paper with double-sided sticky tape and used for parasitization.

Parasitoid. A T. dendrolimi colony was also established by collecting the M. brassicae parasitized eggs from the same field as the host, and maintained for 12 successive generations with eggs of E. kuehniella as a factitious host. Adult wasps which emerged from host eggs were maintained in glass tubes at $25 \pm 0.5^{\circ} \mathrm{C}$, and fed on $10 \%$ honey solution. To avoid cannibalism by hatched larvae, M. brassicae or E. kuehniella eggs were killed by ultraviolet light within $24 \mathrm{~h}$ after oviposition and used for experiments as described by Takada et al. (2000). Female wasps which had mated within $24 \mathrm{~h}$ after emergence were used for parasitization. Parasitization was performed by leaving the female wasps until their death in the glass tube in a conditioned room at $25 \pm 2^{\circ} \mathrm{C}$. The parasitized eggs were reared at $25 \pm 0.5^{\circ} \mathrm{C}$ until emergence of the parasitoid.

Host preference of $\boldsymbol{T}$. dendrolimi after several successive generations. One hundred eggs of each of two different host species, M. brassicae and $E$. kuehniella, were attached to both ends of a strip of paper $(60 \mathrm{~mm}$ in length $\times 10 \mathrm{~mm}$ in width), and placed in a glass tube $(16 \mathrm{~mm}$ in diameter $\times 180 \mathrm{~mm}$ in length). A small piece of paper absorbed with $10 \%$ honey solution was placed in the glass tube as food for the wasp. To exclude the location-effect of food, the wasp's food was placed beside each host's eggs in the glass tube in half of the total replicates. A female wasp, which had mated within $24 \mathrm{~h}$ after emergence, was introduced into the tube. This experiment was performed at the 7 th and 13th generations. Thirteen replicates for the 7th generation and 30 replicates for the 13th generation were prepared. At the 7 th generation, host eggs were exposed to a wasp for $24 \mathrm{~h}$ for parasitization. The number of progeny wasps which emerged from each of the host eggs was counted. Furthermore, for the 13th generation, to determine which of the host species was preferred, the location where the female wasp searched for host eggs was recorded every $30 \mathrm{~min}$ for $3 \mathrm{~h}$ under $25 \pm 0.5^{\circ} \mathrm{C}$. At the 13 th generation it was released for $3 \mathrm{~h}$ for parasitization. To avoid the effect of differences in light intensity in the tube on the behavior of the female, the glass tube was laid parallel to the light for $3 \mathrm{~h}$.

Difference in body size and number of eggs laid by wasps which emerged from two different hosts after 12 successive generations. $T$. dendrolimi, which emerged from the E. kuehniella eggs after 12 successive generations on $E$. kuehniella, were forced to parasitize M. brassicae eggs at the 13th generation. Then, the effect on body size and number of progeny of restoring to the native host after continuous rearing on a factitious host was examined. Each of 25 female wasps which emerged from $M$. brassicae eggs was compelled to parasitize either a $M$. brassicae or an $E$. kuehniella egg at the 14th generation until the wasp's death. Furthermore, each of 25 females emerged from each host was forced to parasitize the respective host's eggs at the 15th generation to ascertain whether or not the restoring effect of the host continued. The number of progeny wasps were counted and their tibia lengths were measured.

Lifetime fecundity of a parasitoid wasp reared on different host species. The mean longevity of adult $T$. dendrolimi females is 5 days (Takada et al., 2000). To clarify the allocated number and sex ratio of progeny in relation to female age, 240 eggs of E. kuehniella were offered to a female wasp daily for 5 days. Parasitized eggs were maintained at $25 \pm 0.5^{\circ} \mathrm{C}$ under continuous daylight. The number of progeny emerging from the parasitized eggs and their sex ratio were examined.

\section{RESULTS}

\section{Host preference of $T$. dendrolimi after 12 contin- uous generation}

In a choice test at the 13th generation, most parasitoid wasps preferred the eggs of $M$. brassicae; two wasps stayed still or searched on the $E$. kuehniella eggs for $3 \mathrm{~h}$ (Fig. 1). Consequently, more progeny emerged from $M$. brassicae eggs than from E. kuehniella eggs. However, no signifi- 


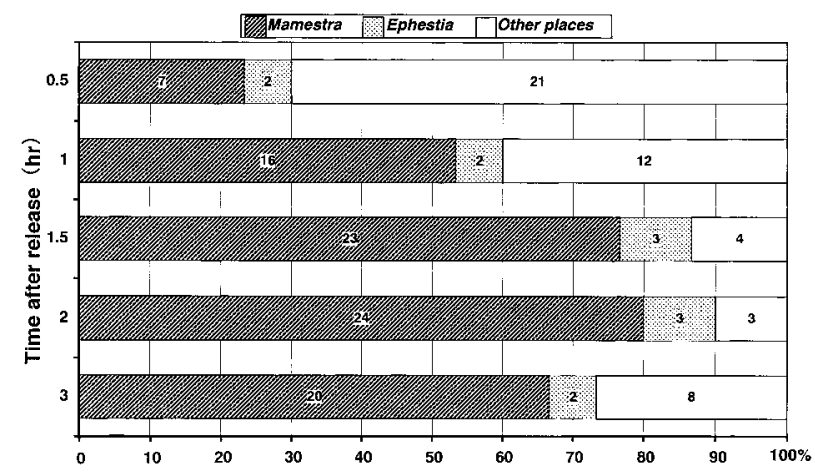

Fig. 1. Location of T. dendrolimi females in a choice test between $M$. brassicae and E. kuehniella eggs. Thirty replicates were observed every $30 \mathrm{~min}$ until $3 \mathrm{~h}$. Dashed bar indicates the number of wasps searching to oviposit on $M$. brassicae host eggs; dotted bar: on E. kuehniella host eggs; and open bar: on other places. There is a significant difference between the three places at $0.5 \mathrm{~h}(0.01<p<0.05)$ and from $1 \mathrm{~h}$ to $3 \mathrm{~h}$ $(p<0.01)$ by $\chi^{2}$ test.

Table 1. Host preference of T. dendrolimi after 12 successive generations on E. kuehniella

$\left.\begin{array}{cccc}\hline \text { Host species } & \begin{array}{c}\text { Number of } \\ \text { wasps } \\ \text { examining } \\ \text { hosts }^{\mathrm{a}}\end{array} & \begin{array}{c}\text { Total number } \\ \text { of progeny } \\ \text { emerged }\end{array} & \begin{array}{c}\text { Number of } \\ \text { progeny-wasps } \\ \text { emerged } \\ \text { per female } \\ \text { (average } \pm \mathrm{SD})\end{array} \\ \hline \text { E. kuehniella } & 3 \mathrm{a} & 79 & 26.3 \pm 5.1 \\ \text { M. brassicae } & 27 \mathrm{~b} & 1,084 & 40.1 \pm 18.4\end{array}\right] \mathrm{NS}^{\mathrm{b}}$

${ }^{\text {a }}$ Thirty replicates were observed. Each 100 eggs of two different hosts, which were attached to both ends of a strip of thick paper, were presented to a female wasp for $3 \mathrm{~h}$. Different letters mean significant difference between the two host species by Binominal test at $p<0.01$.

${ }^{\mathrm{b}}$ No significant difference was observed between the two host species. Statistical analysis was performed by Student- $t$ test $(p=0.2125)$.

cant difference was found in the number of eggs laid per female wasp (Table 1). Similarly, a choice test of the wasps at the 7th generation for the two different host species indicated that more females preferred to oviposit in $M$. brassicae than in $E$. kuehniella eggs, although one female wasp oviposited only in E. kuehniella (Fig. 2).

After $T$. dendrolimi was restored to the native host $M$. brassicae at the 13th generation, the parasitization of E. kuehniella had no effect on hind tibia length (HTL) of progeny wasps between the 14 th and the 15 th generations $(p<0.01$, one-way

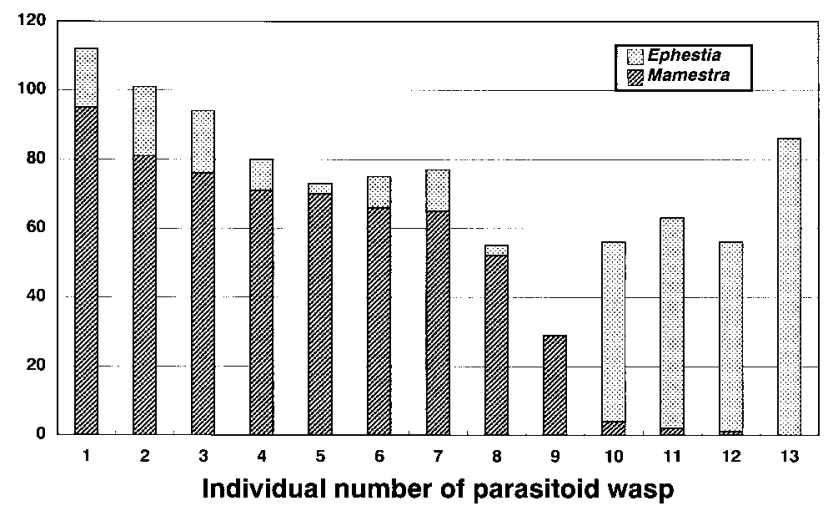

Fig. 2. A choice test of the wasps between M. brassicae and E. kuehniella was performed at the 7 th generation. Each of two host egg clusters was cut off after parasitization for $24 \mathrm{~h}$ and kept at $25 \pm 0.5^{\circ} \mathrm{C}$ until emergence. The number of progeny emerged from each host's eggs was counted. There is a significant difference between E. kuehniella and M. brassicae in all columns $(p<0.01$, Binominal test $)$.

ANOVA) (Table 2). The HTL of $T$. dendrolimi which emerged from $M$. brassicae eggs was longer than that from E. kuehniella eggs at any of the three generations mentioned above. On the other hand, after being restored to the $M$. brassicae eggs T. dendrolimi allocated a greater number of progeny to the E. kuehniella host at the 14th generation, which was almost the same number of progeny as that to the M. brassicae host. However at the 15th generation, the number of progeny from $E$. kuehniella eggs decreased by half (Table 2). These results suggested that the progeny females which emerged from $M$. brassicae have many more eggs than do those from E. kuehniella.

\section{Lifetime fecundity of $T$. dendrolimi reared on different host species}

The longevity of $T$. dendrolimi was 4-5 days. Do the females allocate a similar number of progeny through out the lifetime? Lifetime fecundity differed between the two host species. Female wasps that parasitized $M$. brassicae hosts allocated twice as many progeny as those that parasitized $E$. kuehniella only on the 1st day after emergence (Fig. 3). Two days after emergence the female wasps laid only a small number of eggs regardless of which host species they parasitized (Fig. 3). The rate of female progeny was about $80 \%$ throughout the lifetime of the female wasp regardless of host species (Fig. 4). 
Table 2. Number and hind-tibia length of progeny wasps of T. dendrolimi which emerged from M. brassicae eggs at 13th generation

\begin{tabular}{ccccc}
\hline Host species $^{\mathrm{a}}$ & Generation & $\begin{array}{c}\text { Number of } \\
\text { wasps examined }\end{array}$ & $\begin{array}{c}\text { Hind tibia length } \\
\text { (average } \pm \text { SD } \mu \mathrm{m})\end{array}$ & $\begin{array}{c}\text { Number of progeny } \\
\text { per female } \\
(\text { average } \pm \text { SD) }\end{array}$ \\
\hline M. brassicae & $13 \mathrm{th}$ & 32 & $186.7 \pm 5.1 \mathrm{a}$ & - \\
& $14 \mathrm{th}$ & 25 & $181.2 \pm 11.9 \mathrm{ab}$ & $113.1 \pm 14.8 \mathrm{ab}$ \\
E. kuehniella & $15 \mathrm{th}$ & 25 & $177.2 \pm 11.3 \mathrm{~b}$ & $104.0 \pm 18.4 \mathrm{~b}$ \\
& $14 \mathrm{th}$ & 25 & $140.1 \pm 9.5 \mathrm{c}$ & $128.9 \pm 25.6 \mathrm{a}$ \\
& $15 \mathrm{th}$ & 25 & $134.7 \pm 6.5 \mathrm{c}$ & $54.7 \pm 14.6 \mathrm{c}$ \\
\hline
\end{tabular}

${ }^{\text {a }} T$. dendrolimi which was reared on an E. kuehniella host for 12 continuous generations, parasitized M. brassicae eggs at the 13th generation. Each of 25 wasps which emerged from M. brassicae oviposited in each host species at the 14th generation. Furthermore, each of 25 wasps emerged from each host's eggs parasitized the respective host eggs at the 15th generation.

${ }^{b}$ Different letters mean significant difference on the same column detected by one way ANOVA at 0.01 and by means separation using Fisher's PLSD test (StatView, Abacus Concepts, CA, 1996).

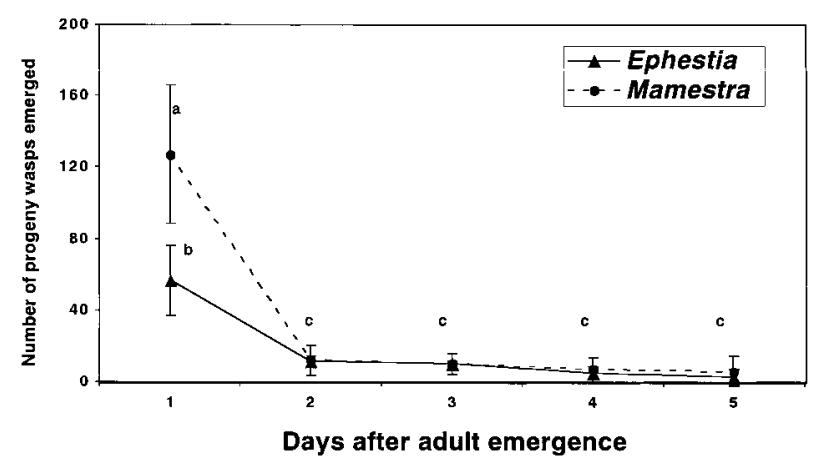

Fig. 3. Change in number of progeny with female aging when M. brassicae (dotted line) or E. kuehniella (solid line) was used as a host.

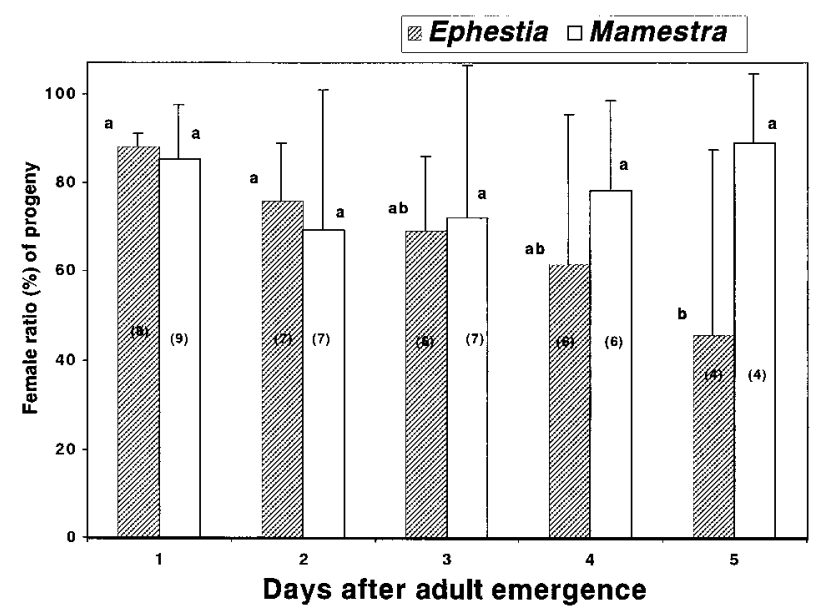

Fig. 4. Sex allocation (female ratio) of progeny wasps which emerged from each host species in relation to female age. There is a significant difference between columns with different alphabetical letters at $p<0.01$ by one way ANOVA with Fisher's PLSD.

\section{DISCUSSION}

$T$. dendrolimi appears to act as an effective control agent of $M$. brassicae populations in cabbage fields maintained without insecticides in Kagamihara, because the parasitization rate of $M$. brassicae egg-masses was $60-80 \%$ and the $M$. brassicae population was kept at a low density for three years (1997 to 1999, unpublished data). When an augmentative biological control program is promoted in cabbage fields, $T$. dendrolimi should be reared continuously over several generations before releasing. However, if host preference for the native host changes, the cabbage field will suffer severe damage.

The finding of this study suggested that host preference of $T$. dendrolimi to $M$. brassicae as a native host did not change even after 12 successive generations on E. kuehniella as a factitious host, although the preference of the parasitoid wasp was not examined when the successive rearing of $T$. dendrolimi, which we obtained from M. brassicae eggs collected in the field was started. However, the facts that three in 30 females preferred the $E$. kuehniella host and never went to a M. brassicae host at the 13th generation (Fig. 1, Table 1), and that some females preferred to oviposit in $E$. kuehniella eggs at the 7th generation (Fig. 2), might indicate a change of host preference. Rojas et al. (1999) reported that after 10 generations of continuous rearing on Callosobruchus maculatus as a factitious host, Catolaccus grandis females switched host acceptance, and 1 generation of rear- 
ing back on the native host, Anthonomus grandis, could not restore acceptance of the natural host to the original level. However, some strains of Trichogramma (including $T$. dendrolimi) did not lose their preference for the native host despite the fact that they were reared on a factitious host for several years (Pavlik, 1993; Lui et al., 1998). Hassan (1989) also reported that 17 strains of $T$. dendrolimi had the highest fecundity and showed a nearly equal preference between the target pests Cydia pomonella, Adoxophyes orana and the factitious host Sitotroga cerealella after rearing for at least 2 generations. Host preference of $T$. dendrolimi may not change easily even after continuous rearing on a factitious host.

E. kuehniella eggs that are used as a factitious host are smaller than M. brassicae eggs. Female wasps emerged from the smaller hosts were less fecund and lived shorter than those from the larger ones (Klomp and Teerink, 1967; Marston and Ertle, 1973; Bigler et al., 1987; Hohmann et al., 1988; Bai et al., 1992; Greenberg et al., 1998b). The size of progeny emerged from the M. brassicae host was larger than that from E. kuehniella among the 13th, 14th and 15th generations (Table 2). When the host species was changed from $M$. brassicae to E. kuehniella, the number of progeny emerging from $E$. kuehniella was not different from that emerged from the $M$. brassicae host (Table 2). These findings suggested that the size of the progeny depended on the host species, and that the female wasps emerging from larger hosts are more fecund than those from smaller ones.

Furthermore, female wasps within 1 day after emergence produced the greatest number of progeny during their lifetime (Fig. 3). Female wasps within 1 day after emergence should, therefore, be used for mass production. On the contrary, this may be a limiting factor when $T$. dendrolimi is mass propagated.

\section{ACKNOWLEDGEMENTS}

The authors wish to express their thanks to Kazuo Hirai for the classification technique of Trichogramma male genitalia and invaluable advice.

\section{REFERENCES}

Bai, B., R. F. Luck, L. Forster, B. Stephens and J. A. M. Jason (1992) The effect of host size on quality attributes of the egg parasitoid, Trichogramma pretiosum. Entomol. Exp. Appl. 64: 37-48.
Bautista, R. and E. J. Harris (1997) Effect of insectary rearing on host preference and oviposition behavior of the fruit fly parasitoid Diachasmimorph longicaudata. Entomol. Exp. Appl. 83: 213-218.

Bergeijk, K. E. van, F. Bigler, N. K. Kaashoek and G. A. Pak (1989) Changes in host acceptance and host suitability as an effect of rearing Trichogramma maidis on a factitious host. Entomol. Exp. Appl. 52: 229-238.

Bigler, F., A. Meyer and S. Bosshart (1987) Quality assessment in Trichogramma maidis Pintureau et Voegele reared from eggs of the factitious hosts Ephestia kuehniella Zell. and Sitotroga cerealella (Oliver). J. Appl. Entomol. 104: 340-353.

Ciochia, V. (1991) Some aspects of the utilization of Trichogramma sp. in Romania. In Trichogramma and Other Egg Parasitoids (E. Wajnberg and S. B. Vinson eds.). Antibes INRA Publ, Paris, pp. 181-182.

Greenberg, S. M., R. K. Morrison, D. A. Nordlund and E. G. King (1998a) A review of the scientific literature and methods for production of factitious hosts for using in mass rearing of Trichogramma spp. (Hymenoptera: Trichogrammatidae) in the former Soviet Union, the United States, western Europe and China. J. Entomol. Sci. 33: $15-32$.

Greenberg, S. M., D. A. Nordlund and Z. Wu (1998b) Influence of rearing host on adult and ovipositional behavior of mass produced female Trichogramma minutum Riley and Trichogramma pretiosum Riley (Hymenoptera: Trichogrammatidae). Biol. Contr. 11: 43-48.

Hamada, R. (1992) Egg parasitoids of common cutworm, Spodoptera litura (Fabricius) (Lepidoptera: Noctuidae). Jpn. J. Appl. Entomol. Zool. 36: 258-259.

Hassan, S. A. (1989) Selection of suitable Trichogramma strains to control the codling moth Cydia pomonella and the two summer fruit tortrix moths Adoxophyes orana, Pandemis heparana [Lep.: Tortricide]. Entomophaga 34: 19-27.

Hassan, S. A., E. Kohler and W. M. Rost (1988) Mass production and utilization of Trichogramma: 10. Control of the codling moth Cydia pomonella and the summer fruit tortrix moth Adoxophyes orana (Lep.: Tortricidae). Entomophaga 33: 413-420.

Hirai, K., Y. Miyakawa and H. Takeuchi (1996) Parasitization efficacy and movement distance by Trichogramma dendrolimi Matsumura on green peppers in a vinylhouse. Proc. Kanto-Tosan Pl. Prot. Soc. 43: 217-219.

Hohmann, C. L., R. F. Luck and E. R. Oatman (1988) A comparison of longevity and fecundity of adult Trichogramma platneri (Hymenoptera: Trichogrammatidae) reared from the eggs of the cabbage looper and the Angoumois grain moth, with and without access to honey. J. Econ. Entomol. 81: 1307-1312.

Klomp, H. and B. J. Teerink (1967) The significance of oviposition rates in the egg parasite, Trichogramma embryophagum Htg. Arch. Neerl. Zool. 17: 350-375.

Lui, S. S., G. M. Zhang and F. Zhang (1998) Factors influencing parasitism of Trichogramma dendrolimi on eggs of the Asian corn borer, Ostrinia furnacalis. Biocontrol 43: 273-287. 
Marston, E. and L. R. Ertle (1973) Host influence of the bionomics of Trichogramma minutum. Ann. Entomol. Soc. Am. 66: 1155-1162.

Nikonov, P. V., G. I. Lebedev and I. P. Startchevsky (1991) Trichogramma production in the USSR. In Trichogramma and Other Egg Parasitoids (E. Wajnberg and S. B. Vinson eds.). Antibes INRA Publ, Paris, pp. 151-152.

Pavlik, J. (1993) Variability in the host acceptance of European corn borer, Ostrinia nubilalis Hbn. (Lep., Pyralidae) in strains of the egg parasitoid Trichogramma spp. (Hym., Trichogrammatidae). J. Appl. Entomol. 115: 77-84.

Rojas, M. G., J. A. Morales-Ramos and E. G. King (1999) Response of Catolaccus grandis (Hymenoptera: Pteromalidae) to its natural host after ten generations of rearing on a factitious host, Callosobrucus maculatus (Coleoptera: Bruchidae). Environ. Entomol. 28: 137-141.

Stinner, R. E. (1977) Efficacy of inundative releases. Annu. Rev. Entomol. 22: 513-531.

Sun, X., Y. Tan and J. Chen (1990) Study of the utilization of Trichogramma dendrolimi to control the masson pine moth Dendrolimus punctatus at longshan forest farm. Forest Res. 3: 407-410.

Takada, Y., S. Kawamura and T. Tanaka (2000) Biological characteristics: growth and development of the egg parasitoid Trichogramma dendrolimi (Hymenoptera: Trichogrammatidae) on the cabbage armyworm Mamestra brassicae (Lepidoptera: Noctuidae). Appl. Entomol. Zool. 35: 369-379. 